\title{
The effect of dose escalation on gastric toxicity when treating lower oesophageal tumours: a radiobiological investigation
}

Rhys Carrington ${ }^{1 *}$, John Staffurth², Samantha Warren ${ }^{3}$, Mike Partridge $^{3}$, Chris Hurt ${ }^{4}$, Emiliano Spezi ${ }^{5}$, Sarah Gwynne ${ }^{6}$, Maria A. Hawkins ${ }^{3}$ and Thomas Crosby ${ }^{1}$

\begin{abstract}
Purpose: Using radiobiological modelling to estimate normal tissue toxicity, this study investigates the effects of dose escalation for concurrent chemoradiation therapy (CRT) in lower third oesophageal tumours on the stomach.

Methods and materials: 10 patients with lower third oesophageal cancer were selected from the SCOPE 1 database (ISCRT47718479) with a mean planning target volume (PTV) of $348 \mathrm{~cm}^{3}$. The original 3D conformal plans $\left(50 \mathrm{G}_{3 \mathrm{D}}\right.$ ) were compared to newly created RapidArc plans of $50 \mathrm{G} \mathrm{y}_{\mathrm{RA}}$ and $60 \mathrm{G} \mathrm{V}_{\mathrm{RA}}$, the latter using a simultaneous integrated boost (SIB) technique using a boost volume, PTV2. Dose-volume metrics and estimates of normal tissue complication probability (NTCP) were compared.
\end{abstract}

Results: There was a significant increase in NTCP of the stomach wall when moving from the 50Gy $\mathrm{V}_{\mathrm{RA}}$ to the 60G $\mathrm{V}_{\mathrm{RA}}$ plans $(11-17 \%$, Wilcoxon signed rank test, $p=0.01)$. There was a strong correlation between the NTCP values of the stomach wall and the volume of the stomach wall/PTV 1 and stomach wall/PTV2 overlap structures $(R=0.80$ and $R=0.82$ respectively) for the 60G $\mathrm{y}_{\mathrm{RA}}$ plans.

Conclusion: Radiobiological modelling suggests that increasing the prescribed dose to 60Gy may be associated with a significantly increased risk of toxicity to the stomach. It is recommended that stomach toxicity be closely monitored when treating patients with lower third oesophageal tumours with 60Gy.

\section{Introduction}

The incidence of lower third oesophagus tumours are increasing in most Western populations [1] and it is becoming increasingly clear that chemo-radiotherapy (CRT) is now a valid alternative to surgical resection in the treatment of both oesophageal and gastroesophageal junction (GEJ) cancer [2] \& [3]. It has been shown that a combined approach results in a significantly higher overall survival rate compared to either chemotherapy or radiotherapy alone [4] \& [5]. However, local in-field recurrence is still the main reason of treatment failure [6] following definitive CRT, with $>75 \%$ of these occurring within the gross tumour volume (GTV) when the standard radiation dose of $\approx 50 \mathrm{~Gy}$ is delivered. Indeed, local

\footnotetext{
* Correspondence: carringtondr@cardiff.ac.uk

'School of Medicine, Cardiff University, Cardiff, UK

Full list of author information is available at the end of the article
}

recurrence also contributes towards a worse prognosis in GEJ carcinoma [3].

In theory, a higher radiation dose delivered to the tumour should result in higher local control rate. However it is only with the recent technological advances in radiotherapy (RT) planning and delivery that the ability to deliver increased dose to the tumour whilst minimising dose to normal, healthy tissue and organs at risk (OAR) is becoming possible [7]. Increased tumour control probability (TCP) should therefore be achievable by increasing the standard dose prescription beyond $\approx$ 50Gy. A retrospective study by Zhang et al. [8] found that there was significantly higher overall survival in their patient cohort if the patient was treated in a high dose group ( $>51 \mathrm{~Gy}$ ) or a low dose group (<51Gy), whilst Geh et al. found there was a dose-response relationship between increasing prescribed radiotherapy dose and pathological complete response [9]. Bedford et al. [10] also found that conformal techniques offered the potential of a 
5-10Gy increase in dose delivered to the GTV up to 60Gy with acceptable increases in toxicity.

The organs most at risk when planning oesophageal radiotherapy treatment, and for which the most stringent dose constraints are usually applied are the heart, lungs and spinal cord. Oesophageal cancer cases will therefore be planned according to a combination of the achievable dose coverage of the planning treatment volume (PTV) and the meeting of dose constraints for these organs. The SCOPE 1 study has shown low rates of acute and late toxicity with CRT using 4 cycles of cisplatin and capecitabine, with cycles three and four given concurrently with 50 Gy in 25 fractions of radiotherapy [11]. However, the 24 week failure free survival was significantly better in the CRT only arm than the CRT plus cetuximab arm $(76.9 \%$ (90\% confidence interval 69 . $7-83 \cdot 0)$ vs $66 \cdot 4 \%,(58 \cdot 6-73 \cdot 6))$ and cetuximab will therefore not be carried forward in future clinical trials. Work by this group in preparation for the forthcoming SCOPE 2 trial [12] has shown that dose escalation to 62.5Gy in mid oesophageal patients is feasible, with the additional dose able to be delivered without exceeding the OAR dose constraints in $75 \%$ of patients. However, dose escalation has not yet been studied in lower oesophageal cancers, when the added proximity of the relatively radiosensitive stomach provides an added planning challenge [13]. With the role of radiotherapy dose escalation identified as a research priority [14] for improving outcomes, it is important to quantify the increased risk that this may pose in sites such as the lower oesophagus where clinical evidence for dose-toxicity correlation for adjacent organs (such as stomach) is lacking. This planning study therefore aims to investigate the feasibility of lower oesophageal dose escalation with a focus on toxicity to the stomach.

\section{Methods and materials}

10 patients with tumours in the lower region (centre of tumour at $32-40 \mathrm{~cm}$ from back of teeth measured via Endoscopic ultrasound (EUS)) were selected at random from both arms of the SCOPE 1 database and their classification as lower region tumours confirmed visually. SCOPE1 has been ethically approved by the Research Ethics Committee for Wales and has approval from the Medicines and Health Care Product Regulatory Agency to be conducted in the UK. The subset had a range of planning target volumes (PTV1) from 219 to $484 \mathrm{~cm}^{3}$ and a mean volume of $348 \mathrm{~cm}^{3}$, similar to that of the entire SCOPE 1 cohort (mean $327 \mathrm{~cm}^{3}$ ). The GTVs and OARs outlined as per the SCOPE protocol were re-used.

PTV 1 is grown by adding $1 \mathrm{~cm}$ isotropcially to the clinical treatment volume (CTV), itself grown by adding $1 \mathrm{~cm}$ radially and $2 \mathrm{~cm}$ superiorly and inferiorly (along axis of oesophagus) to the GTV and may include the stomach mucosa at the inferior limit. For the purpose of this specific study and the use of the simultaneous integrated boost (SIB) technique for dose escalation, additional structures were also created. A PTV2 (boost volume) was created for the dose escalated plans by adding an isotropic $0.5 \mathrm{~cm}$ margin to the GTV, supported by a study by Hawkins et al. [15] and reflecting the technique in the SCOPE 2 trial where margins will not be adjusted dependent on tumour position [12]. The protocol did not address stomach filling or any dose constraints for that organ specifically. There were no constraints or protocol concerning the filling state of the stomach in the SCOPE 1 trial and therefore for the patients in this study. The stomach was contoured as (a) whole organ and (b) stomach wall. The stomach wall volume was generated by creating a ring like structure encompassing the outer $5 \mathrm{~mm}$ of the whole stomach outline. This has been shown to provide a satisfactory approximation of stomach wall thickness [16] \& [17]. In addition, the stomach and stomach wall structures were divided into the volume that was within PTV1 (StomachIn and StomachWall-In) and outside PTV1 (Stomach-Out and StomachWall-Out). Specific dose constraints were given for each for the SIB plans (Table 1) based on the recommendations of the Quantitative Analyses of Normal Tissue Effects in the Clinic (QUANTEC) paper for dose volume effects in the stomach and small bowel [18]. An SIB dose of 60Gy in 25 fractions was considered to be clinically meaningful and is being taken forward within an ongoing prospective dose escalation trial (SCOPE 2).

Table 1 Dose constraints for radiotherapy plans

\begin{tabular}{|c|c|}
\hline \multicolumn{2}{|l|}{ Dose Constraints } \\
\hline \multicolumn{2}{|c|}{ Dose-volume constraints } \\
\hline \multirow[t]{2}{*}{ PTV1 (50 Gy) } & $V_{95 \%}(47.5 \mathrm{~Gy})>95 \%$ \\
\hline & $D_{\max }(0.1 \mathrm{cc})<107$ \% (53.5 Gy) \\
\hline \multirow[t]{2}{*}{ PTV2 (60 Gy) } & $V_{95 \%}(57 \mathrm{~Gy})>95 \%$ \\
\hline & $D_{\max }(0.1 \mathrm{cc})<107 \%$ (64.2 Gy) \\
\hline \multirow[t]{2}{*}{ Lung } & Mean dose < 20 Gy \\
\hline & $V_{20 G y}<25 \%$ \\
\hline \multirow[t]{3}{*}{ Heart } & Mean dose $<25$ Gy \\
\hline & $V_{30 G y}<45 \%^{a}$ \\
\hline & $V_{40 G y}<30 \%{ }^{b}$ \\
\hline CordPRV & $D_{\max }(0.1 \mathrm{cc})<40$ Gy (45 Gy permitted) \\
\hline Liver & $V_{30 G y}<60 \%$ \\
\hline Individual Kidneys & $V_{20 G y}<25 \%$ \\
\hline Stomachln ${ }^{c}$ & Max dose <60Gy \\
\hline StomachOut $^{c}$ & Max dose < 45Gy \\
\hline
\end{tabular}

${ }^{a}$ Applies only to $50 \mathrm{~Gy}_{\mathrm{RA}}$ and $60 \mathrm{G} \mathrm{y}_{\mathrm{RA}}$ plans

${ }^{\mathrm{b}}$ Applies only to $50 \mathrm{~Gy} \mathrm{y}_{3 \mathrm{D}}$ plans

${ }^{c}$ Applies only to $60 \mathrm{~Gy} \mathrm{y}_{\mathrm{RA}}$ plans 
All treatment planning was undertaken in Eclipse version 10 (Varian, Palo Alto CA). The original 3D conformal plans were imported in DICOM format and the doses recalculated using the AAA algorithm with a $2.5 \mathrm{~mm}$ grid. RapidArc (RA) plans were generated using 2 arcs of $360^{\circ}$, clockwise and counter-clockwise with a collimator rotation of $\pm 10^{\circ}$. The 50Gy 3D conformal plans $\left(50 \mathrm{~Gy}_{3 \mathrm{D}}\right.$ ) were then compared to 50Gy RapidArc plans $\left(50 \mathrm{~Gy}_{\mathrm{RA}}\right)$ and to plans with an additional simultaneously integrated boost of 60Gy to PTV2 (60Gy $\mathrm{GA}_{\mathrm{RA}}$ ) (See Fig. 1). Dose constraints are listed in Table 1 and additional dose-volume metrics were calculated for each structure (Table 2). Patient 6 was originally planned using $50 \mathrm{~Gy}_{\mathrm{RA}}$ therefore a $50 \mathrm{~Gy}$ 3D plan was not created in this case.

Radiobiological modelling of TCP was undertaken using the parameters derived by Geh et al. [9]. This multivariate logistics regression model was constructed using data from 26 pre-operative CRT trials in oesophageal cancer and was considered a good representative of the SCOPE 1 patient cohort. The TCP modelling was undertaken bin-wise in Microsoft Excel using and parameters by Geh et al. found in their original paper [9]. Differential dose-volume histograms $(\mathrm{DVH})$ for each structure were calculated in CERR utilising Matlab scripts developed in-house [19] before being converted to relative DVHs in Microsoft Excel. TCP was calculated as:

$$
T C P(z)=\frac{\exp (z)}{1+\exp (z)}
$$

where $\mathrm{z}=\mathrm{a}_{0}+\mathrm{a}_{1}$ total RT dose $+\mathrm{a}_{2}$ total RT dose $\times$ dose per fraction $+a_{3}$ duration $+a_{4}$ age $+a_{5} 5$ FU dose $+a_{6}$ cisplatin dose. The $\alpha / \beta$ was $4.9 \mathrm{~Gy}$.

Normal tissue complication probability (NTCP) modelling was carried out in Eclipse Biological Evaluation module using the whole heart volume model of Gagliardi et al. [20] and for the lung using the model parameters from De Jaeger et al. [21], which predicts a radiation pneumonitis (RP) of grade 2 or higher. NTCP models for the stomach are limited therefore modelling was carried out using those judged to be most relevant. The whole stomach was modelled using parameters derived by Burman et al. [22] with the endpoint being ulceration, whilst the stomach wall parameters were derived by Feng et al. [23], modelling the probability of $\geq 3$ grade gastric bleeding.

Data were analysed using the SPSS statistics package version 20.0.0 (IBM), and results are reported as median (range) values. Both the $\mathrm{Z}$-score and the $P$-Values were calculated.

\section{Results}

Table 2 reports the dose-volume metrics and the results of the Wilcoxon signed rank test for all radiotherapy plans. Adequate target dose coverage was possible for all
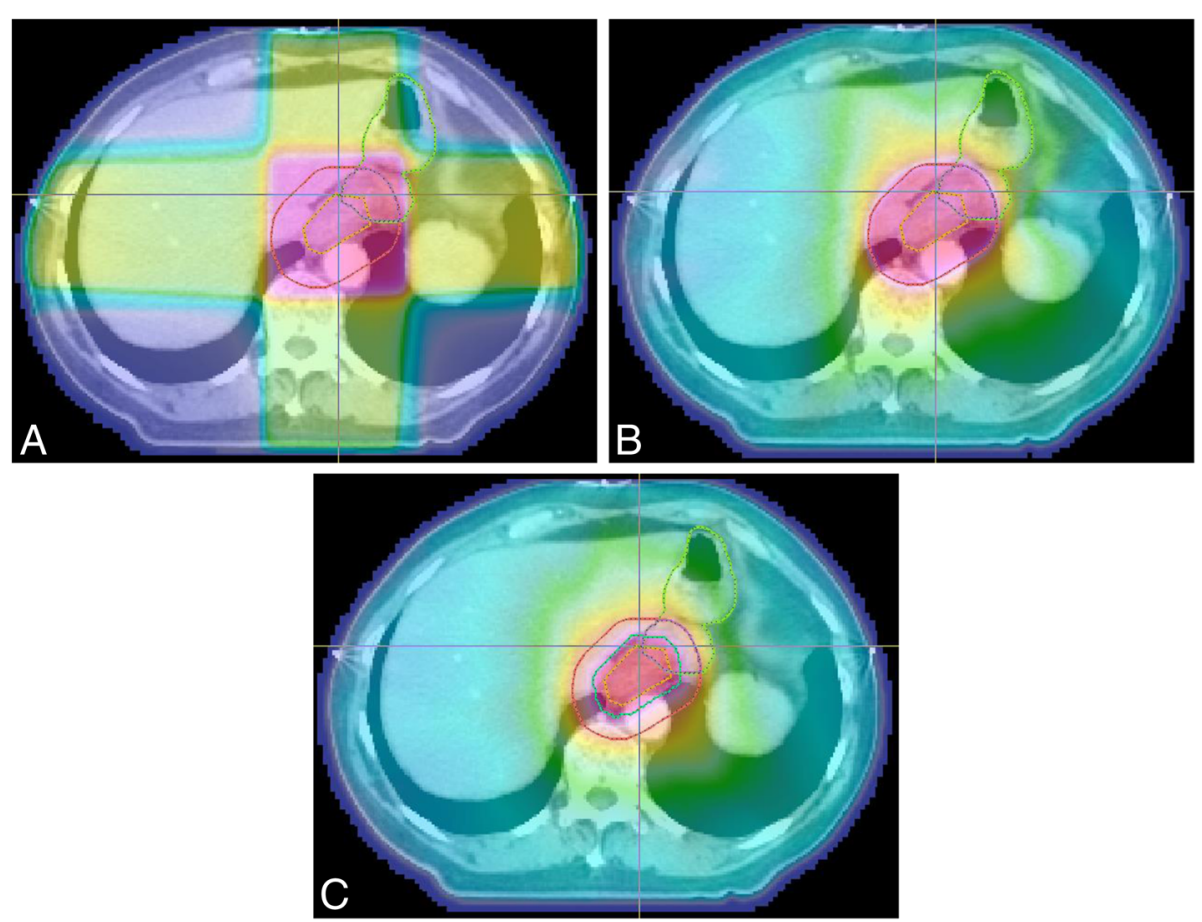

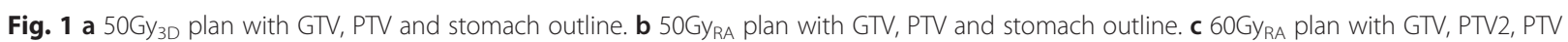
and stomach outline. Outlines: GTV- dashed orange, PTV- dashed red, PTV2- dashed blue, Stomach- dashed green 
Table 2 Dose volume metrics for all radiotherapy plans

\begin{tabular}{|c|c|c|c|c|c|c|}
\hline \multicolumn{6}{|c|}{ Comparison of dose-volume metrics, TCP and NTCP values } & \multirow[b]{3}{*}{$50 G y_{R A}-60 G y_{R A}$} \\
\hline & \multirow{2}{*}{$\begin{array}{l}50 G y_{3 D} \\
\text { Median (range) }\end{array}$} & \multirow{2}{*}{$\begin{array}{l}50 G y_{R A} \\
\text { Median (range) }\end{array}$} & \multirow{2}{*}{$\begin{array}{l}\text { 60Gy } \\
\text { Median (range) }\end{array}$} & \multicolumn{2}{|c|}{ Wilcoxon signed-rank test } & \\
\hline & & & & $50 G y_{3 D}-50 G y_{R A}$ & $50 G y_{3 D}-60 G y_{R A}$ & \\
\hline \multicolumn{7}{|l|}{ PTV1 } \\
\hline V95\% & $98.2(96.0-100)$ & $99.1(95.2-100)$ & $97.0(95.0-98.2)$ & $Z=0.53(p=.57)$ & $Z=1.07(p=.28)$ & $Z=1.36(p=.17)$ \\
\hline \multicolumn{7}{|l|}{ PTV2 (GTV + $0.5 \mathrm{~cm})$} \\
\hline V95\% & & & $95.1(92.4-97.4)$ & & & \\
\hline TCP (\%) Geh & $38.7(37.5-41.1)$ & $37.8(37.5-38.7)$ & $50.9(50.7-51.4)$ & $Z=2.11(p=.04)$ & $Z=2.67(p=.01)$ & $Z=2.81(p=.01)$ \\
\hline \multicolumn{7}{|l|}{ Lung } \\
\hline Mean dose (Gy) & $9.8(6.0-11.1)$ & $10.2(5.8-14.3)$ & $10.7(6.4-15.2)$ & $Z=1.78(p=.07)$ & $Z=2.40(p=.02)$ & $Z=2.80(p=.01)$ \\
\hline V13Gy (\%) & $26.8(20.0-35.9)$ & $32.8(15.1-51.6)$ & $34.4(18.0-54.2)$ & $Z=2.19(p=.03)$ & $Z=2.55(p=.01)$ & $Z=2.09(p=.04)$ \\
\hline V20Gy (\%) & $19.7(12.3-24.3)$ & $11.3(4.6-17.4)$ & $15.6(6.5-23.4)$ & $Z=2.55(p=.01)$ & $\mathrm{Z}=1.72(p=.09)$ & $Z=2.81(p=.01)$ \\
\hline NTCP (\%) De Jaeger & $5.1(1.9-6.0)$ & $4.3(2.8-8.0)$ & $4.7(3.1-9.0)$ & $Z=1.49(p=.14)$ & $Z=2.09(p=.04)$ & $Z=2.80(p=.01)$ \\
\hline \multicolumn{7}{|l|}{ Heart } \\
\hline Mean dose (Gy) & $26.8(13.9-31.2)$ & $21.2(14.6-23.6)$ & $20.2(16.4-23.2)$ & $Z=1.68(p=.09)$ & $Z=1.58(p=.11)$ & $Z=0.15(p=.88)$ \\
\hline V30Gy (\%) & $55.1(9.7-67.9)$ & $17.2(8.2-25.3)$ & $18.7(10.3-22.6)$ & $Z=2.67(p=.01)$ & $Z=2.55(p=.01)$ & $Z=0.87(p=.39)$ \\
\hline V40Gy (\%) & $16.2(5.9-24.5)$ & $10.1(4.5-14.8)$ & $10.6(5.6-13.6)$ & $Z=2.67(p=.01)$ & $Z=2.67(p=.01)$ & $Z=1.58(p=.11)$ \\
\hline NTCP (\%) Gagliardi & $8.9(3.1-12.8)$ & $4.9(2.2-7.3)$ & $6.1(2.9-7.9)$ & $Z=1.90(p=.06)$ & $Z=1.38(p=.17)$ & $Z=2.80(p=.01)$ \\
\hline \multicolumn{7}{|l|}{ Stomach } \\
\hline Mean dose (Gy) & $29.8(5.5-44.2)$ & $24.1(5.4-40.4)$ & $23(6.5-36.1)$ & $Z=1.17(p=.24)$ & $Z=0.97(p=.33)$ & $Z=1.60(p=.11)$ \\
\hline Max dose (Gy) & $52.6(49.6-53.4)$ & $51.9(42.4-52.9)$ & $60.9(51.6-61.6)$ & $Z=0.83(p=.41)$ & $Z=2.61(p=.01)$ & $Z=2.81(p=.01)$ \\
\hline V45 (cc) & $47.3(7.3-80.4)$ & $32.8(0-49.8)$ & $34.3(5.4-25.4)$ & $Z=2.60(p=.01)$ & $Z=2.50(p=.01)$ & $Z=0.36(p=.72)$ \\
\hline V50 (cc) & $31.5(0-23.4)$ & $17.7(0-14.8)$ & $21.4(2.2-19.2)$ & $Z=2.31(p=.02)$ & $Z=1.78(p=.07)$ & $Z=1.27(p=.20)$ \\
\hline Stomachln max dose (Gy) & $52.6(49.6-53.4)$ & $51.9(42.4-52.9)$ & $60.9(51.6-61.6)$ & $Z=0.77(p=.44)$ & $Z=2.61(p=.01)$ & $Z=2.81(p=.01)$ \\
\hline StomachOut max dose (Gy) & $51.4(49.4-53.1)$ & $44.4(36.6-43.6)$ & $44.8(42.3-46.1)$ & $Z=1.76(p=.07)$ & $Z=1.79(p=.07)$ & $Z=0.14(p=.88)$ \\
\hline NTCP (\%) Burman & $0.6(0-2.5)$ & $0.2(0-1.3)$ & $0.3(0-3.4)$ & $\mathrm{Z}=2.38(p=.02)$ & $Z=0.35(p=.73)$ & $Z=2.03(p=.04)$ \\
\hline \multicolumn{7}{|l|}{ Stomach wall } \\
\hline Mean dose (Gy) & $29.5(8.2-42.6)$ & $22.9(7.9-38.7)$ & $22.4(9.1-35.0)$ & $\mathrm{Z}=0.97(p=.33)$ & $Z=0.76(p=.45)$ & $Z=0.87(p=.39)$ \\
\hline Max dose (Gy) & $52.6(49.6-53.4)$ & $51.9(43.4-52.9)$ & $61(51.6-61.6)$ & $Z=0.77(p=.44)$ & $Z=2.55(p=.01)$ & $Z=2.81(p=.01)$ \\
\hline V45 (cc) & $28(6.2-39.9)$ & $17.9(0-26.9)$ & $17.9(5.4-25.4)$ & $Z=2.19(p=.03)$ & $Z=2.19(p=.03)$ & $Z=0.46(p=.65)$ \\
\hline V50 (cc) & $15.8(0-23.4)$ & $9.1(0-14.8)$ & $9.2(2.2-19.2)$ & $Z=2.31(p=.02)$ & $Z=1.48(p=.14)$ & $Z=1.28(p=.20)$ \\
\hline NTCP (\%) Feng & $17.4(3.5-24.9)$ & $11.1(3.6-18.9)$ & $17.5(3.2-39.4)$ & $Z=1.72(p=.09)$ & $Z=1.99(p=.05)$ & $Z=2.70(p=.01)$ \\
\hline \multicolumn{7}{|l|}{ Cord PRV } \\
\hline Dmax 0.1 cc (Gy) & $36.9(16.1-41.3)$ & $31.1(26.2-44.1)$ & $34.9(28.4-39.6)$ & $Z=0.47(p=.64)$ & $Z=0.18(p=.86)$ & $Z=1.67(p=.10)$ \\
\hline
\end{tabular}

patients in all treatment modalities when considering the coverage of PTV1 (Table 2). 4 patients failed to meet the minimum coverage of PTV2 with the minimum coverage being $92.4 \%$. All OAR dose for the heart and lung were met for all patients for all treatment plans. 6 patients failed to meet the Stomach-In constraint and 1 failed to meet the Stomach-Out constraint for the 60GyA plans. All other dose constraints in Table 1 were met.

There was a mean decrease $1.0 \%(-3.0 \%, 0.6 \%)$ in TCP from the $50 \mathrm{~Gy}_{3 \mathrm{D}}$ to the $50 \mathrm{~Gy}$ RA plans, a mean increase of $12.0 \%(9.9 \%, 13.6 \%)$ in TCP from the $50 \mathrm{~Gy}_{3 \mathrm{D}}$ plans to the $60 \mathrm{~Gy} y_{\text {RA }}$ plans and a mean increase of
$13.0 \%(12.4 \%, 13.4 \%)$ in TCP from the $50 \mathrm{~Gy}_{\mathrm{RA}}$ plans to the $60 \mathrm{~Gy}_{\text {RA }}$ plans. For NTCP there was a mean decrease of $3.4 \%(-6.3 \%, 0 \%)$ for the heart from the $50 \mathrm{~Gy}_{3 \mathrm{D}}$ to the $50 \mathrm{~Gy}$ RA plans, a mean decrease of $2.2 \%$ $(-4.9 \%, 2.0 \%)$ from the $50 \mathrm{~Gy}_{3 \mathrm{D}}$ to the $60 \mathrm{~Gy} \mathrm{R}_{\mathrm{RA}}$ plans and a mean increase of $1.2 \%(0.5 \%, 2.0 \%)$ in NTCP for the heart from the $50 G y_{R A}$ to the $60 G y_{R A}$ plans. For lung there was a mean increase of $0.4 \%(-0.8 \%, 2.2 \%)$ in NTCP from the $50 \mathrm{~Gy}_{3 \mathrm{D}}$ to the $50 \mathrm{~Gy}_{\mathrm{RA}}$ plans, a mean increase of $1.0 \%(-0.6 \%, 3.2 \%)$ from $50 \mathrm{~Gy}_{3 \mathrm{D}}$ to $60 \mathrm{~Gy}_{\mathrm{RA}}$, and a mean increase of $0.6 \%(0.1 \%, 1.2 \%)$ from the $50 \mathrm{~Gy} \mathrm{y}_{\mathrm{RA}}$ to the $60 \mathrm{Gy_{RA }}$ plans. 
For the stomach and stomach wall the variation in NTCP between patients was considerable. Patients 1, 2, $6 \& 8$ all had stomach NTCP values $<0.03 \%$ for all treatment plans whilst the largest value was $3.4 \%$ for a patient planned using the $60 \mathrm{~Gy} y_{\mathrm{RA}}$ technique. The stomach wall model, which models a different endpoint, showed considerably larger absolute values of NTCP, the largest being $39.4 \%$ for a patient treated with the $60 \mathrm{~Gy} y_{R A}$ plan. Across the whole study, there was a mean decrease in stomach wall NTCP of $3.1 \%(-6.5,0 \%)$ from the $50 \mathrm{~Gy}_{3 \mathrm{D}}$ plans to the $50 \mathrm{~Gy}_{\mathrm{RA}}$ plans, a mean increase of $5.9 \%(-4.7,18.7 \%)$ in NTCP from the $50 \mathrm{~Gy}_{3 \mathrm{D}}$ to the 60 Gy $y_{\text {RA }}$ plans and a mean increase of $8.2 \%(-0.4$, $21.3 \%$ ) in NTCP from the $50 G y_{\text {RA }}$ to the $60 \mathrm{~Gy}$ RA plans (NTCP values see Fig. 2).

When the NTCP modelling is restricted to the volume outside the boost volume (PTV2), there was in general a smaller difference between the NTCP values between plans. In this case there was a mean decrease of $3.4 \%$ $(-7.4 \%, 0.3 \%)$ from the $50 \mathrm{~Gy}_{3 \mathrm{D}}$ to the $50 \mathrm{~Gy}$ RA plans, a mean decrease of $0.9 \%(-4.7 \%, 1.0 \%)$ in NTCP from the $50 \mathrm{~Gy}$ B to the $60 \mathrm{~Gy}_{\mathrm{RA}}$ plans, and a mean increase of $2.3 \%(-0.4 \%, 6.9 \%)$ in NTCP from the $50 \mathrm{~Gy}_{\mathrm{RA}}$ to the $60 \mathrm{~Gy}_{\text {RA }}$ plans (Fig. 3).

Table 3 shows the Pearson correlation coefficients between the Stomach and Stomach Wall volumes and associated dose metrics. It can be seen how the strongest correlations are between the stomach wall volumes in each plan and the mean does received by those volumes $\left(0.63,0.66\right.$ and 0.66 for the $50 \mathrm{~Gy}_{3 \mathrm{D}}, 50 \mathrm{~Gy}_{\mathrm{RA}}$ and $60 \mathrm{~Gy} \mathrm{RA}_{\mathrm{RA}}$ respectively).

Six patients had an overlap between the GTV and PTV2 and Stomach Wall structure whilst all patients had an overlap between the PTV1 and Stomach Wall structures. There was a strong correlation between the
NTCP value and the Stomach Wall structure/PTV1 overlap structure volume for all treatment plans (Pearson's $R=0.80,0.77$ and 0.77 for the $60 G y_{R A}, 50 G y_{R A}$ and $50 \mathrm{~Gy}$ (D plans respectively). Fig. 4 shows the correlation between NTCP and the Stomach Wall/PTV1 overlap structure volume for the $60 \mathrm{~Gy} y_{\mathrm{RA}}$ plans.

There was also a strong correlation between the NTCP value and the Stomach Wall/PTV2 overlap structure volume for the $60 \mathrm{~Gy}$ RA plan $(R=0.82)$ (Fig. 5).

\section{Discussion}

This study has shown that using the SIB technique it is possible to deliver a dose of 60Gy to the tumour whilst adhering to all standard OAR dose constraints for lower oesophagus tumours.

It is acknowledged that the TCP model used in this investigation does not account for Cetuximab administration, however Cetuximab will not be administered in the SCOPE 2 trial at which this study is aimed. A strength of the TCP model proposed by Geh et al. is that it combines a diverse range of trials, and it was therefore considered the most appropriate to use here. It has been shown that there is a small reduction $(<1 \%)$ in TCP when comparing the $50 \mathrm{~Gy}$ D plans to the $50 \mathrm{~Gy} \mathrm{~V}_{\mathrm{RA}}$ plans. There was a higher lung mean V13Gy, but reduced V20Gy, Heart V30 /40Gy, Stomach V45/50 cc and Stomach Wall V45/50 cc. When comparing the 50Gy $y_{\text {RA }}$ to the $60 \mathrm{~Gy}$ RA plans there was a significant increase in TCP but also an increase in the mean dose parameter for the lung (See Table 2). There was a significant increase in mean TCP $(\approx 12)$ going from the $50 G y_{3 D}$ to the $60 \mathrm{~Gy}$ RA plan. Comparing 50Gy 3 and $50 \mathrm{~Gy} y_{\mathrm{RA}}$, there was a statistically significant increase in lung V13Gy, which can be explained by the low dose wash associated with RapidArc type treatment plans, however V20Gy reduced

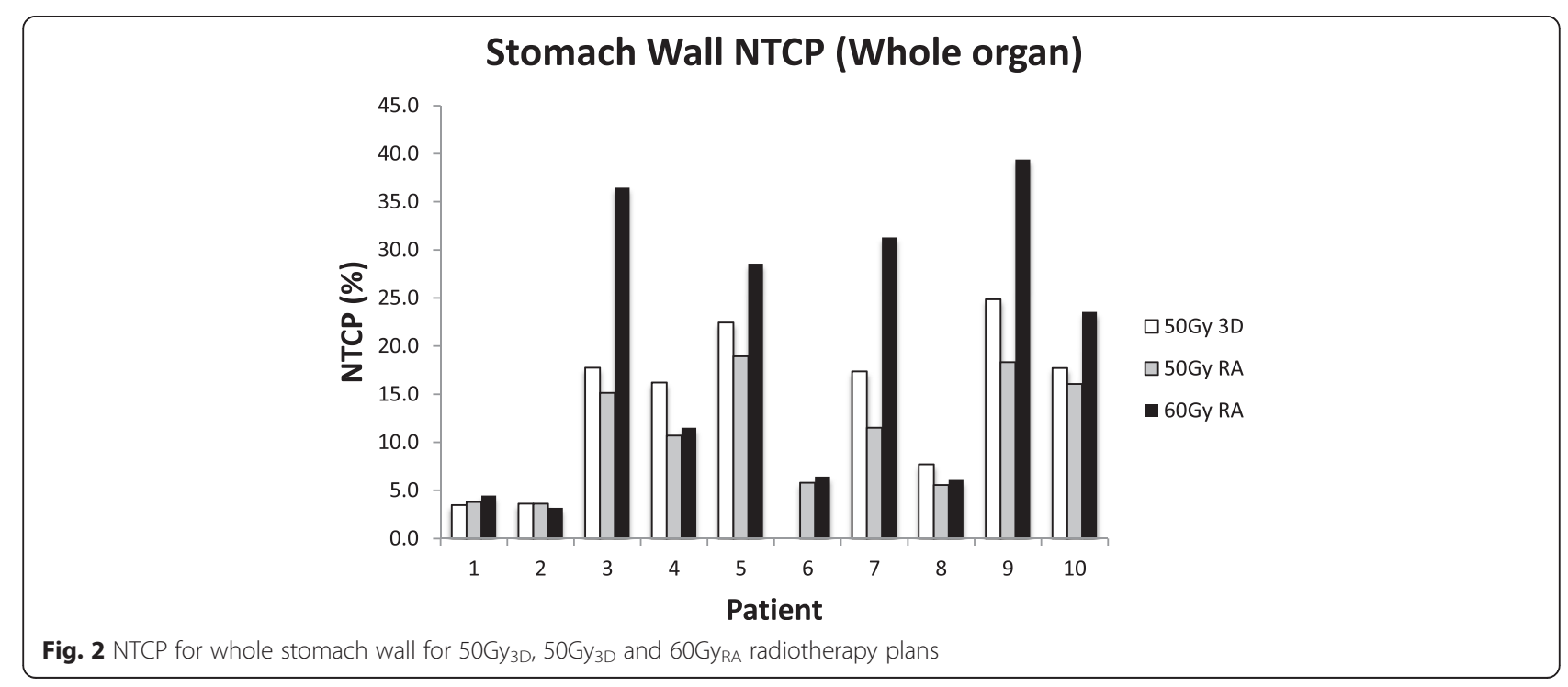




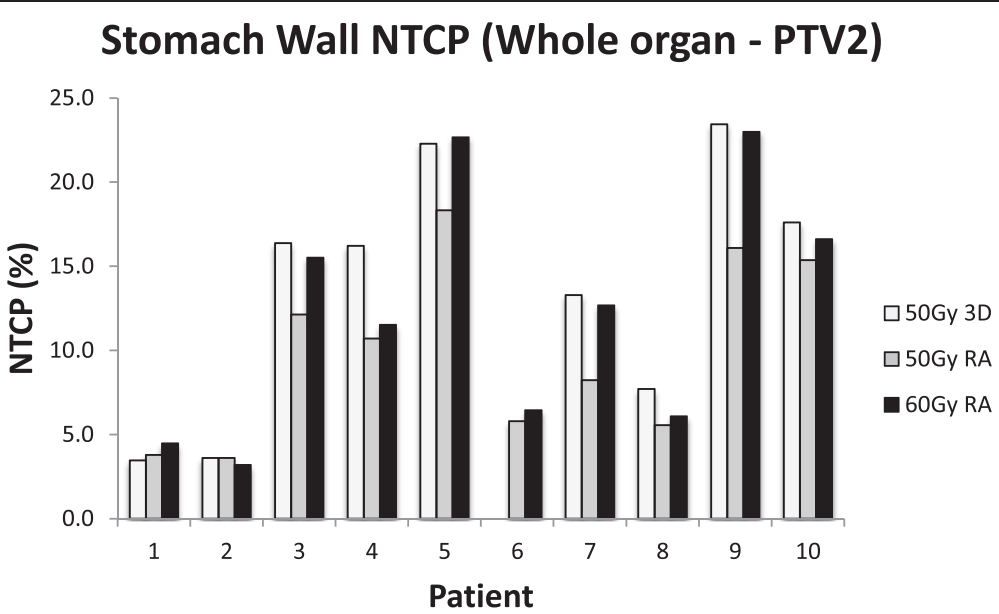

Fig. 3 NTCP for stomach wall minus PTV2 for 50Gy $y_{3 D}, 50 G y_{3 D}$ and $60 G y_{R A}$ radiotherapy plans

and mean lung NTCP was reduced from $5.1 \%$ to $4.3 \%$. There was a significant decrease in the heart V30/40Gy values. Although this did not result in a significant decrease in NTCP between the two planning methods in this study, this agrees with results from our previous work on mid-oesophageal cancer patients [12].

Moving from the $50 \mathrm{~Gy}_{\mathrm{RA}}$ to the $60 \mathrm{~Gy} \mathrm{y}_{\mathrm{RA}}$ plans, although the NTCP values for the heart and lung were lower than those found in our previous study on mid oesophageal cancer patients as would be expected, there was still a similar modest increase in heart and lung toxicities when using the boost technique [12]. This also agrees with the recently published study by Roeder et al. who delivered 60Gy to patients with oesophageal cancer using a SIB technique and found acceptable acute and late overall toxicity to the lung and heart [24]. However, when treating lower oesophageal tumours there is the added complication of having the stomach adjacent to the treatment volume. The involvement of the group in a proposed randomized clinical trial investigating dose escalation (SCOPE 2) therefore led to this study, which is the first to specifically investigate the effect of dose escalation in lower oesophageal tumours on the stomach using radiobiological modelling. It is acknowledged that biological modelling and the resulting outcomes are extremely dependent on the model parameters used as well as how they are applied. As a result, we used two models for the stomach and applied them to both to the structure as a whole and inside and outside the PTV. The model for the stomach wall by Feng et al. [23] was found to predict a higher rate of toxicity than that for the whole stomach, most likely the result of the different endpoints of gastric bleeding and ulceration being modelled respectively. Max dose constraints of 45Gy and 60 Gy were applied to the stomach outside (StomachOut) and inside (Stomach-In) the PTV respectively for the $60 \mathrm{~Gy}_{\mathrm{RA}}$ plans. The NTCP results for the $60 \mathrm{~Gy} \mathrm{PA}_{\mathrm{RA}}$ when modelling the volume outside the PTV were similar to those of the $50 \mathrm{~Gy}_{\mathrm{RA}}$ and $50 \mathrm{~Gy}_{3 \mathrm{D}}$ plans (Max NTCP of $23.0 \%$ and $23.4 \%$ for the $60 \mathrm{~Gy}_{\mathrm{RA}}$ and $50 \mathrm{~Gy}_{3 \mathrm{D}}$ plans respectively), suggesting that dose escalation may not pose any more risk to normal stomach than 3D conformal radiotherapy (Fig. 3). However, when considering the stomach wall structure as a whole it was found that

Table 3 Pearson correlation coefficients between stomach, stomach wall volumes and dose metrics

\begin{tabular}{lccc}
\hline & \multicolumn{2}{c}{ Pearson Coefficient } & 50GyRA \\
\cline { 2 - 3 } & 50Gy3D & 0.60 & 0.12 \\
\hline Stomach Volume - Stomach Mean Dose & 0.35 & 0.08 & 0.61 \\
Stomach Volume - Stomach Max Dose & -0.19 & 0.05 & 0.55 \\
Stomach Volume - Stomach V45 & 0.16 & 0.66 \\
Stomach Volume - Stomach V50 & 0.11 & 0.02 \\
Stomach Wall Volume - Stomach Wall Mean Dose & 0.63 & 0.32 & 0.66 \\
Stomach Wall Volume - Stomach Wall Max Dose & -0.12 & 0.21 & 0.68 \\
Stomach Wall Volume - Stomach Wall V45 & 0.23 & 0.22 & 0.12 \\
Stomach Wall Volume - Stomach Wall V50 & 0.38 & 0.04 \\
\hline
\end{tabular}




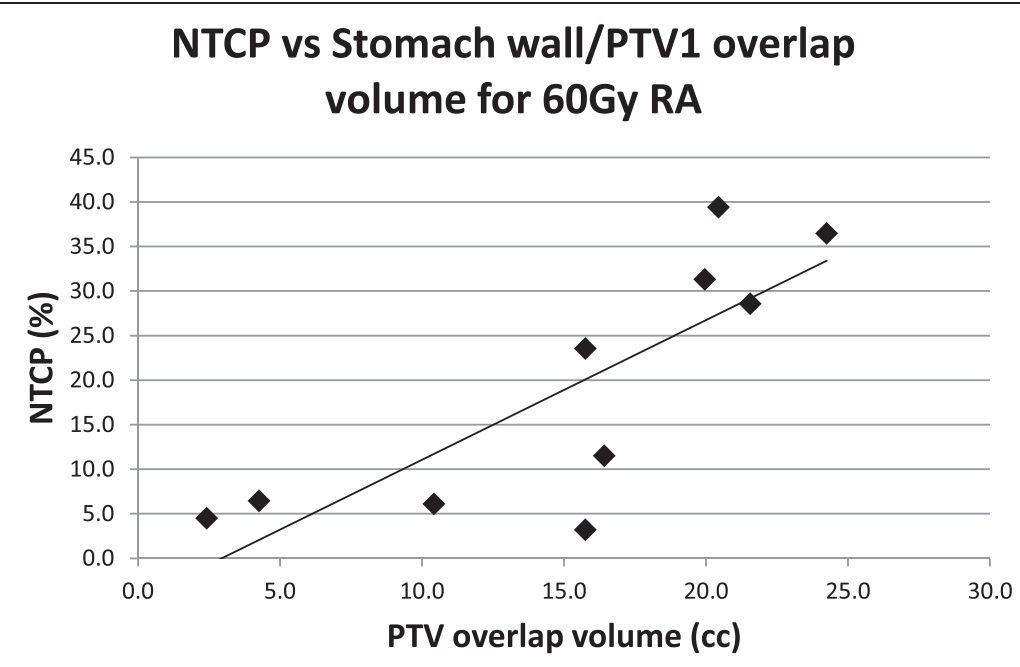

Fig. 4 NTCP vs whole stomach wall/PTV1 overlap structure volume for $60 \mathrm{~Gy}_{\mathrm{RA}}$ radiotherapy plans

there was up to $20 \%$ increase in NTCP when using the dose escalation plan compared to the $50 \mathrm{~Gy}_{\mathrm{RA}}$ plan. This value however could be considered as being the worse case scenario, as it is acknowledged that stomach movement and filling over the course of treatment may blur out any dose hot spots. The analysis of any accompanying Cone Beam CT data of these patients would help quantify this movement however this data was unavailable. Any NTCP value is also by nature calculated from a model that is open to interpretation therefore should only be used to give an approximate risk. It is fully acknowledged that radiobiological modelling inherently has limitations that limit its accuracy. Specifically in the case of this study, there is a lack of both clinical outcome data and radiobiological models for stomach toxicity when prescribing a dose $>50 \mathrm{~Gy}$. However the model used was deemed to be the most suitable in this instance. The application of radiobiological modelling to partial organ irradiation is also a contentious one that may affect the results. However the purpose of this study was not to give definitive values of stomach toxicity, but to investigate and inform of the potential relative risks involved in dose escalation of lower esophageous tumours both in a forthcoming trial and in clinical practice.

We have shown that there is a strong correlation in NTCP with the volume of overlap between the stomach wall with both PTV1 and the high dose region PTV2. When more clinical data is available it may become apparent that safe delivery of the 60Gy SIB is dependent on this volume of the overlap, which could potentially be reduced by reducing the treatment margins for

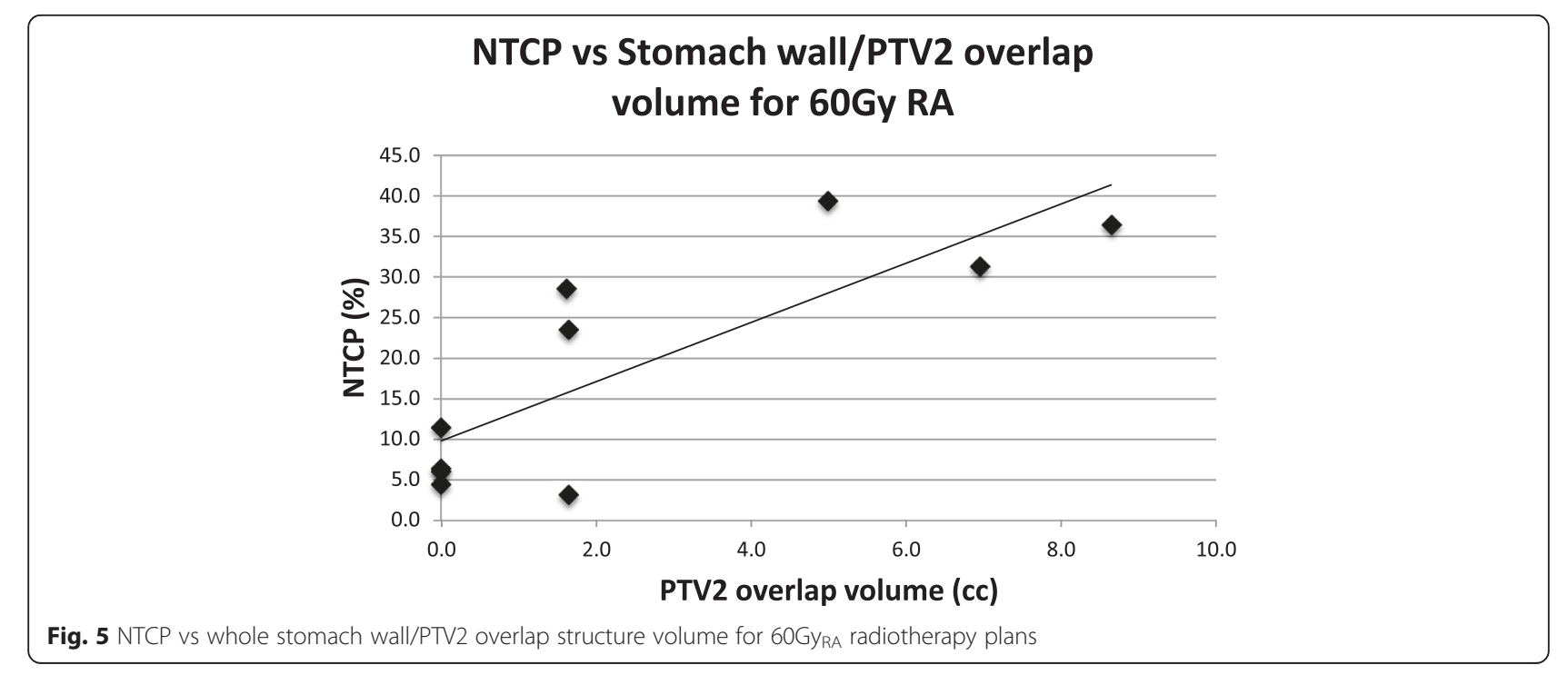


individual patients using techniques such as $4 \mathrm{DCT}$, gating and breath hold protocols. However it has been reported that the inter-patient motion of oesophageal tumours is highly variable [25] and that even the use of 4DCT may not even fully account for organ motion in between fractions [26]. Nakamura et aldiscuss how large variations in stomach volume may have a detrimental effect on dose escalation when treating pancreatic cancer, despite using a breath hold technique [27]. The impact of variation in gas in the stomach on dose distribution should also be considered. For example, Kumagai et al. found that dose conformation to the CTV was degraded due to bowel gas movement when treating pancreatic cancer using carbon ion beams [28] and consequently may also be applicable when using photon beams. Bouchard et al. also found that changes in stomach filling resulted in the boost target being missed when treating GEJ tumours with IMRT-SIB [29]. A move to reduction in populationbased margins from those used in the SCOPE 1 and SCOPE 2 trials, rather than on an individual basis, may therefore increase the risk of failure to control the disease. The margins used in this investigation were taken from the SCOPE 2 protocol therefore give an approximation of results from a forthcoming nationwide trial, taking into account the inherent errors in radiobiological modelling.

Concerning the impact of stomach filling, as there was no stomach filling protocol for the SCOPE 1 trial an area for further work would; be to investigate what impact, if any, the inclusion of a filling or breath hold protocol would have on stomach toxicity and dose distribution when treating lower oesophageal tumours. However this is beyond the scope of this current work as it would require either the incorporation of a protocol into a clinical trial made available for analysis or a retrospective analysis of patients who underwent an appropriate strategy prior to treatment.

An inclusion criteria for the SCOPE 1 trial was that patients were to have histologically confirmed carcinoma of the oesophagus with no more than $2 \mathrm{~cm}$ of mucosal tumour extension into the stomach. As this patient group is likely to be included in the SCOPE 2 trial, this study's findings mean it is likely that it be advised in the radiotherapy protocol that these patients be treated with caution until the safety of this dose escalation method is clearly defined within the SCOPE 2 trial.

The results of this study also suggest that the maximum prescribed dose achievable for each patient may be dependent on the volume of the stomach overlap with the treatment volume. Further work will therefore include modelling individualised dose prescription.

\section{Conclusion}

Radiobiological modelling suggests that increasing the prescribed dose to 60Gy may be associated with a significantly increased risk of toxicity to the stomach within the boost volume. The results of this study also suggest that the maximum prescribed dose safely achievable for each patient in the future may be dependent on the volume of the stomach within the treatment volume. It is recommended that stomach toxicity be closely monitored prospectively when treating patients with lower oesophageal tumours in the forthcoming SCOPE 2 trial.

\section{Abbreviations}

CRT: Chemo-radiotherapy; CTV: Clinical Treatment Volume; DVH: Dose Volume Histogram; EUS: Endoscopic Ultrasound; GEJ: Gastroesophageal Junction; GTV: Gross Tumour Volume; NTCP: Normal Tissue Complication Probability; OAR: Organ At Risk; PRV: Planning Risk Volume; PTV: Planning Treatment Volume; RA: RapidArc; RT: Radiotherapy; SIB: Simultaneous Integrated Boost; TCP: Tumour Control Probability.

\section{Competing interests}

The authors declare that they have no competing interests.

\section{Authors' contributions}

RC Carried out planning study and drafting of manuscript. JS Involved in drafting the manuscript and revising it critically for important intellectual content. SW Involved in conception and design of study and drafting of manuscript. MP Involved in conception and design of study and drafting of manuscript. CH Substantially involved in acquisition of trial data. ES Involved in drafting the manuscript and revising it critically for important intellectual content. SG Involved in drafting the manuscript and revising it critically for important intellectual content. MH Involved in conception and design of study and drafting of manuscript. TC Involved in acquisition of data and drafting of manuscript. All authors read and approved the final manuscript.

\section{Acknowledgments}

Rhys Carrington received a PhD studentship grant from Cancer Research Wales. Grant number: 2445.

Dr Warren and Dr Partridge are supported by Cancer Research UK. Grant number: C5255/A15935.

Dr Hawkins is supported by MRC grant MC_PC_12001/2.The authors are grateful to Cardiff University (Orca) for funding this open access publication.

\section{Author details}

${ }^{1}$ School of Medicine, Cardiff University, Cardiff, UK. ${ }^{2}$ Department of Clinical Oncology, Velindre Cancer Centre, Cardiff, UK. ${ }^{3}$ CRUK MRC Oxford Institute for Radiation Oncology Gray Laboratories, University of Oxford, Old Road Campus Research Building, Oxford, UK. ${ }^{4}$ Wales Cancer Trials Unit, Cardiff University, Cardiff, UK. ${ }^{5}$ School of Engineering, Cardiff University, Cardiff, UK. ${ }^{6}$ South West Wales Cancer Centre, Swansea, UK.

Received: 9 February 2015 Accepted: 4 November 2015 Published online: 19 November 2015

\section{References}

1. Botterweck AA, Schouten LJ, Volovics A, Dorant E, Van Den Brandt PA. Trends in incidence of adenocarcinoma of the oesophagys and gastric cardia in ten European countries. Int J Epidemiol. 2000;29(4):645-54.

2. Teoh AYB, Chiu PWY, Yeung WK, Liu SYW, Wong SKH, Ng EKW. Long-term survival outcomes after definitive chemoradiation versus surgery in patients with resectable squamous carcinoma of the esophagus: results from a randomized controlled trial. Ann Oncol. 2013;24(1):165-71.

3. Buergy D, Lorh F, Baack T, Siebenlist K, Haneder S, Michaely H, et al. Radiotherapy for tumours of the stomach and gastroesophageal junction a review of its role in multimodal therapy. Radiat Oncol. 2012;7:192.

4. Cunningham D, Starling N, Rao S. Capecitabine and oxaliplatin for advanced esophagogastric cancer. N Engl J Med. 2008;358:36-46.

5. Herskovic A, Martz K, Al-Sarraf M, Leichman L, Brindle J, Vaitkevicius V, et al. Combined Chemotherapy and Radiotherapy Compared with Radiotherapy Alone in Patients with Cancer of the Esophagus. N Engl J Med. 1992;326(24):1593-8. 
6. Settle SH, Bucci MK, Palmer MB, Liu H, Liengsawangwong R, Guerrero TM, et al. PET/CT fusion with treatment planning $C T$ (TP CT) shows predominant pattern of locoregional failure in esophagea patients treated with chemoradiation in in GTV. Int J Radiat Oncol Biol Phys. 2008;72:S72-S3.

7. Fakhrian K, Oechsner M, Kampfer S, Schuster T, Molls M, Geinitz H. Advanced techniques in neoadjuvant radiotherapy allow dose escalation without increased dose to organs at risk. Strahlenther Onkol. 2013;189:293-300.

8. Zhang Z, Liao Z, Jin J, Ajani J, Chang JY, Jeter M, et al. Dose-response relationship in locoregional control for patients with stage II-III esophageal cancer treated with concurrent chemotherapy and radiotherapy. Int J Radiat Oncol Biol Phys. 2005;61(3):656-64.

9. Geh JI, Bond SJ, Bentzen SM, Jones RG. Systematic overview of preoperative (neoadjuvant) chemoradiotherapy trials in oesophageal cancer: Evidence of a radiation and chemotherapy dose response. Radiother Oncol. 2006;78:236-44.

10. Bedford JL, Viviers L, Guzel Z, Childs PJ, Webb S, Tait DM. A quantitive treatment planning study evaluating the potential of dose escalation in conformal radiotherapy of the oesophagus. Radiother Oncol. 2000;57:183-93.

11. Hurt G, Nixon L, Griffiths G, Al-Mokhtar R, Gollins S, Staffurth J, et al. SCOPE1: a randomised phase $\| / / I I$ multicentre clinical trial of definitive chemoradiation, with or without cetuximab, in carcinoma of the oesphagus. BMC Cancer. 2011;11:466-78.

12. Warren S, Partridge M, Carrington R, Hurt C, Crosby T, Hawkins M. Radiobiological determination of dose escalation and normal tissue toxicity in definitive chemoradiation therapy for esophageal cancer. Int J Radiat Oncol Biol Phys. 2014;90:423-9.

13. Rubin P, Casarett GW. Clin Radiat Pathol. Vol 1. 1968. W.B. Saunders Co, 1st Edition.

14. Gwynne S, Falk S, Gollins S, Wills L, Bateman A, Cummins S, et al. Oesophageal Chemoradiotherapy in the UK - A Current Practive and Future Directions. Clin Oncol. 2013;25:368-77.

15. Hawkins MA, Aitken A, Hansen VN, McNair HA, Tait DM. Set-up errors in radiotherapy for oesophageal cancers - Is electronic portal imaging or conebeam more accurate? Radiother Oncol. 2011;98(2):249-54.

16. Rapaccini GL, Aliotta A, Pompili M, Grattagliano A, Anti M, Merlino B, et al. Gastric wall thickness in normal and neoplastic subjects: a prospective study performed by abdominal ultrasound. Gastrointest Radiol. 1988;13(3):197-9.

17. Pickhardt PJ, Asher DB. Wall thickening of the gastric antrum as a normal finding: multidetector $C T$ with cadaveric comparison. AJR Am J Roentgenol. 2003;181(4):973-9.

18. Kavanagh BD, Pan CC, Dawson LA, Das SK, Allen X, Haken RKT, et al. Radiation Dose-Volume Effectes in the Stomach and Small Bowel. Int J Radiat Oncol Biol Phys. 2010;76(3):S101-S7.

19. Gwynne S, Spezi E, Wills L, Nixon L, Hurt C, Joseph G, et al. Toward semi-automated assessment of target volume delineation in radiotherpay trials: the SCOPE 1 pretrial test case. Int J Radiat Oncol Biol Phys. 2012:84(4):1037-42.

20. Gagliardi G, Lax I, Ottolenghi A, Rutqvist LE. Long-term cardiac mortality after radiotherapy of breast cancer - application of the relative seriality model. Br J Radiol. 1996;69(825):839-46.

21. De Jaeger K, Hoogeman MS, Engelsman M, Seppenwoolde Y, Damen EMF, Mijnher BJ, et al. Incorporatin an improved dose-calculation algorithm in conformal radiotherapy of lung cancer: re-evaluation of dose in normal lung tissue. Radiother Oncol. 2003;69(1):1-10.

22. Burman C, Kutcher GJ, Emami B, Goitein M. Fitting of normal tissue tolerance data to an analytic function. Int J Radiat Oncol Biol Phys. 1991;21(1):123-5.

23. Feng M, Normolle D, Pan CC, Dawson LA, Amarnath S, Ensminger WD, et al. Dosimetric Analysis of Radiation-Induced Gastric Bleeding. Int J Radiat Oncol Biol Phys. 2012;84(1):e1.

24. Roeder F, Nicolay NH, Nguyen T, Ebrahimi LS, Askoxylakis V, Bostel T, et al. Intensity modulated radiotherapy (IMRT) with concurrent chemotherapy as definitive treatment of locally advanced esophageal cancer. Radiat Oncol. 2014;9:191.

25. Lever FM, Lips IM, Crijns SPM, Reerink O, van Lier ALHMW, Moerland MA, et al. Quantification of Esophageal Tumor Motion on Cine-Magnetic Resonance Imaging. Int J Radiat Oncol Biol Phys. 2014;88(2):419-24.

26. Wang J, Lin SH, Dong L, Balter P, Mohan R, Komaki R, et al. Quantifying the Interfractional Displacement of the Gastroesophageal Junction during Radiation Therapy for Esophageal Cancer. Int J Radiat Oncol Biol Phys. 2012;83(2):e273-e80.
27. Nakamura A, Shibuya K, Nakamura M, Matsuo Y, Shiinoki T, Nakata M, et al. Interfractional dose variations in the stomach and the bowels during breathhold intensity-modulated radiotherapy for pancreatic cancer: Implications for a dose-escalation strategy. Med Phys. 2013;40(2):021701.

28. Kumagai M, Hara R, Mori S, Yanagi T, Asakura H, Kishimoto R, et al. Impact of Intrafractional Bowel Gas Movement on Carbon Ion Beam Dose Distribution in Pancreatic Radiotherapy. Int J Radiat Oncol Biol Phys. 2009;73(4):1276-81.

29. Bouchard M, McAleer MF, Starkchall G. Impact of gastric filling on radiation dose delivered to gastroesophageal junction tumours. Int J Radiat Oncol Biol Phys. 2010;77:292-300.

\section{Submit your next manuscript to BioMed Central and take full advantage of:}

- Convenient online submission

- Thorough peer review

- No space constraints or color figure charges

- Immediate publication on acceptance

- Inclusion in PubMed, CAS, Scopus and Google Scholar

- Research which is freely available for redistribution 